\title{
Performance of a Communicating Radar using FSK and Fractional Fourier Transform for Automotive Applications
}

\author{
Pasquale Striano, Christos V. Ilioudis, Carmine Clemente, and John J. Soraghan \\ Centre for Signal and Image Processing, University of Strathclyde, Glasgow, United Kingdom \\ pasquale.striano, c.ilioudis, carmine.clemente, j.soraghan- @ strath.ac.uk
}

\begin{abstract}
In automotive environment, it is preferable to have a joint radar and communication system as it is able to use the same hardware and band of the spectrum for both systems. In this paper a joint radar communication system for automotive radar based on the Fractional Fourier Transform (FrFT) is presented. The performance of the system is analysed in the presence of interference arising from other users. In addition, the communication performance in terms of Bit Error Rate (BER), is analysed for different scenarios. Simulation results demonstrate the potential of the proposed system in multi-user scenarios and in different types of communication channels.
\end{abstract}

Index Terms - Radar, Communication, Automotive, FrFT, FSK.

\section{INTRODUCTION}

In the past decade, many researchers have focussed on the identification of technical and technological solutions to create smart vehicular environments. Several researches investigated the design of safer roads in which vehicles are able to share road state information, such as the presence of an accident and possible detours.

Given the increasing number of automotive radars, the issue of interferences must be dealt with. Specifically for the radar, the reception of interfering signals can lead to problems such as ghost targets. The problem is present on both the radar and the communication sides in a communicating radar system. An efficient and effective solution to the spectrum share problem is the use of joint radar-communication techniques, which principal idea is to use the same transceiver for radar and communication purposes.

In [1], [2], the authors presented a joint radarcommunication system based on Orthogonal FrequencyDivision Multiplexing (OFDM) for automotive application, which is able to reach a very high bit rate of $20 \mathrm{Mb} / \mathrm{s}$.

In [3] the Fractional Fourier Transform (FrFT) was shown to be suitable for orthogonal waveforms generation for MIMO radar systems, while in [4] a joint radarcommunication system based on FrFT designed waveform was presented. This system does not fit the standards of automotive radar and communication as it employs pulsed waveforms instead of Continuous Waveform (CW), which is more suitable for short range applications [5].

In [6] the authors presented a joint radar-communication system based on FrFT for automotive radar. The wave- forms are transmitted in a consecutive manner, similar to the Frequency modulation with rapid chirps [7].

This paper extends [6] focusing on the detection performance in a scenario where two users share the same frequency band with small changes in the used FrFT order and on the communication performance in different types of propagation channels. The remaining of the paper has the following structure. Section II describes the system, while in Section III the detection performance is assessed in the presence of interference caused by other users. In Section IV the communication performance is presented assuming that the system experiences slow-flat fading channels. Finally, Section V concludes the paper.

\section{THE FRACTIONAL FOURIER TRANSFORM}

The Fractional Fourier Transforms (FrFTs) belongs to the class of linear time-frequency representations (TFRs) that have been extensively used by the signal processing community. The FrFT has been introduced by Namias in 1980 [8] and it is a generalization of the Fourier Transform (FT). The FrFT is a linear operation that corresponds to the rotation of the signal along the axis $u$ making an angle $\phi$ with $u^{\prime}$ axis. It has been used in a wide rage of applications such as the waveform propagation, filter design, signal analysis and pattern recognition. Letting $x\left[u^{\prime}\right]$ be an arbitrary signal of length $U$, its $\alpha$-order discrete FrFT is defined as [8]:

$$
\mathbf{X}_{\alpha}[u]=\sum_{u^{\prime}=-U / 2}^{U / 2} K_{\alpha}\left[u, u^{\prime}\right] x\left[u^{\prime}\right]
$$

where $\alpha$ is the fractional transform order, corresponding the rotation angle $\phi=\alpha \frac{\pi}{2}$ and $K_{\alpha}\left[u, u^{\prime}\right]$ is the FrFT kernel function defined in [8]. In the proposed approach the FrFT is used as multiplexing tool that maps bits of information in chirp-like signals, as in [6].

\section{WAVEFORM DESIGN}

The block diagram of the mono-static radar in the considered basic configuration of the FrFT based joint radar and communication system is shown at the top of Fig.1. As it can be seen, $B$ bits of information are generated from a source and for $B$ bits, $G$ guard bits are 


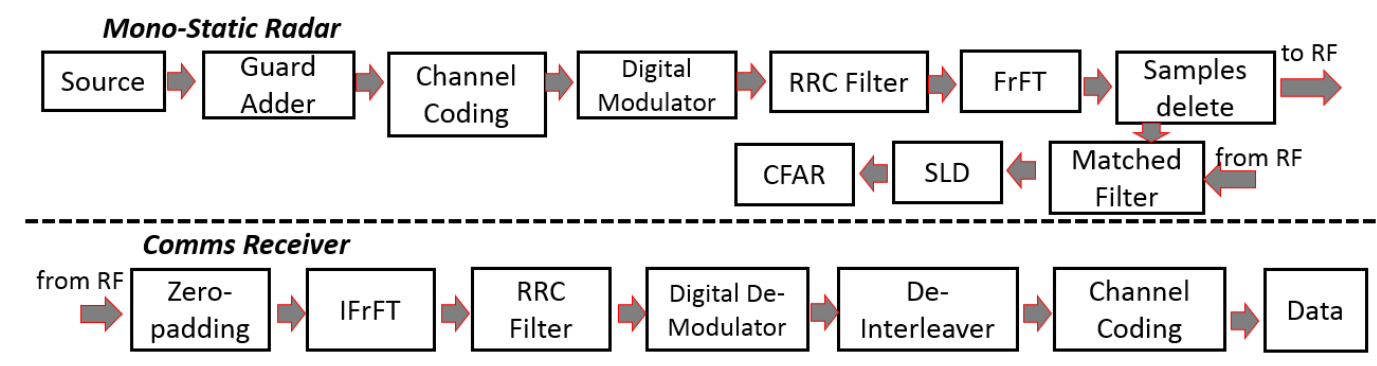

Fig. 1: Block diagram of (top) the mono-static radar and (bottom) the Communication Receiver of the FrFT based on a joint radar-communication basic configuration

added at the end of the sequence in order to compensate the group delay introduced by the Root Raised Cosine (RRC) filter. Then the sequence is spread by using a $L$-long Barker code, leading to a coded sequence of $(B+G) \times L$ bits. The interleaver ${ }^{1}$ is used for burst error-correction. It is applied only to the $B \times L$ bits of information and aims to spread a burst of errors across the entire spread sequence. The digital modulator is the Frequency-Shift Keying (FSK) in which digital information is transmitted through discrete frequency changes of a carrier signal. This modulator assigns $N_{s}$ number of samples per symbol and for each symbol assigns a frequency band $f_{\text {sep }}$, leading to $(B+G) \times L \times N_{s}$ long symbol sequence. The RRC filter is used to minimise the Inter-Symbol Interference (ISI) that may be caused by the channel. For efficiency, it is implemented as a multirate filter that up-samples the output by a factor $R_{s}$, leading to a final sequence of $U=$ $(B+G) \times L \times N_{s} \times R_{s}$ samples. The sequence obtained after applying the RRC filter is then mapped into a chirplike waveform by applying an FrFT with a specific order $\alpha$. After applying the FrFT, to keep the transmitted power at near constant level and to get a FMCW (Frequency Modulated Continuous Waveform), the waveform goes through a sample remove process as investigated in [6].

During the detection process, the radar acquires the echoes and performs a matched filter with the transmitted waveform. After the matched filter a Square Law Detector (SLD) is applied. Furthermore, to perform the detection the magnitude of each waveform is compared with a conventional Cell Averaging Constant False Alarm Rate (CA-CFAR) [9].

\section{A. Receiver}

In Fig. 1 the block diagram of the communication receiver is illustrated. The length of the input of the Inverse FrFT (IFrFT) must be the same length of the signal after the FrFT in transmission. For this reason a zeropadding is applied at the beginning and at the end of the received signal. The zero-padding leads to a sequence of

\footnotetext{
${ }^{1}$ The interleaver is applied to mitigate the impact of burst of errors in scenarios with strong fading.
}

$(B+G) \times L \times R_{s} \times N_{s}$ samples, that enters in the IFrFT block to perform an inverse rotation of that applied in the radar transmitter. The output signal is the passed through the RRC filter, which also down-samples the waveform by a factor $R_{s}$. The digital demodulator translates the $(B+G) \times L \times N_{s}$ long sequence of symbols in a sequence of $(B+G) \times L$ bits, according to the modulation employed. At this point, the de-interleaver performs the inverse of the interleaver. The chip correlator block correlates the input spread sequence with the $L$-long Barker code used in transmission to extract $B$ bits, exploiting both the low correlation side lobes and the knowledge that the peaks of the correlation occurs every $L$ samples. In this way the $B$ bits of information can be recovered.

\section{Detection Performance}

In this section, the detection performance of the proposed system is evaluated in the presence of two radars that are in close proximity to each other and operate in the same frequency band. The two users are uniquely identified from two different orders of the FrFT. When the difference, $\alpha_{\text {diff }}$, between two orders of the FrFT decreases there will be an increase of mutual interference. Consequently there will be a worsening of the detection performance caused from the presence of ghost targets.

The ratio of the radar's useful reflected signal to the interfering signal power is signal-to-interference ratio (SIR) and is given by:

$$
\mathrm{SIR}=\frac{P_{\mathrm{obj}}}{P_{\mathrm{Int}}}=\frac{R_{\mathrm{Int}}^{2} \sigma_{\mathrm{obj}}}{4 \pi R_{\mathrm{obj}}^{4}}
$$

where $P_{\mathrm{obj}}$ is the power of the reflected radar signal from the car, computed by the radar equation, $P_{\mathrm{Int}}$ is the power of user interference attenuated by the channel, computed using the Friis equation, $R_{\mathrm{Int}}$ and $R_{\mathrm{obj}}$ are the

TABLE I: Simulated Scenario Parameter

\begin{tabular}{clc}
\hline Parameters & Description & Values \\
\hline$R_{\mathrm{obj}}$ & Distance from the target. & $100 \mathrm{~cm}$ \\
\hline$R_{\mathrm{Int}}$ & Distance of interference users. & $80 \mathrm{~cm}$ \\
\hline$\sigma_{\mathrm{obj}}$ & Radio Cross Section (RCS). & $10 \mathrm{dBm}^{2}$ \\
\hline
\end{tabular}




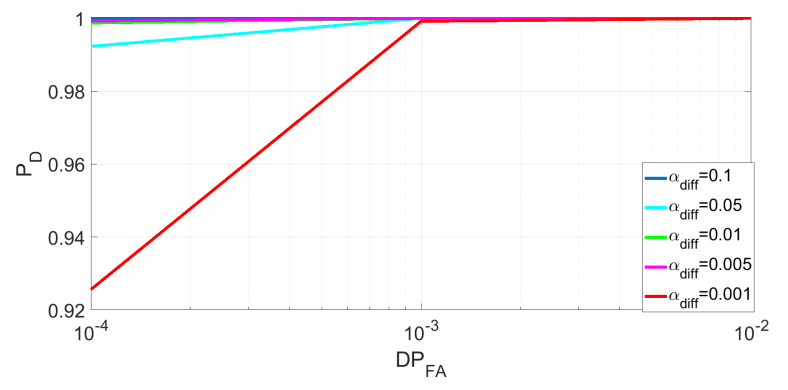

Fig. 2: Receiver Operating Characteristic (ROC) curves between $P_{D}$ and design $P_{F A}$.

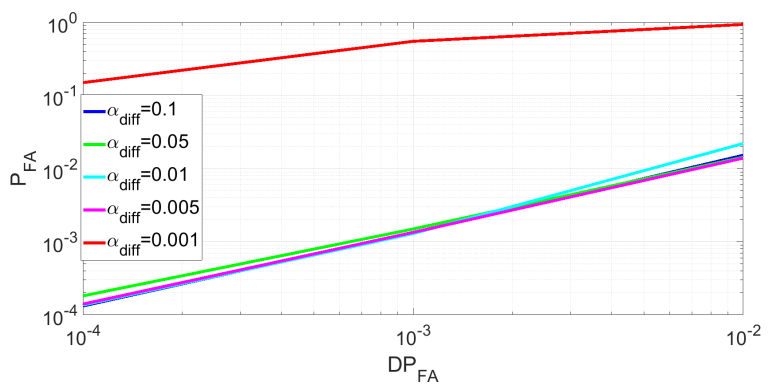

Fig. 3: $P_{F A}$ values versus design $D P_{F A}$.

distances of interference user and the object from main user respectively. Using the parameters of the scenario in Table I the SIR is $-13 \mathrm{~dB}$.

The detection performance is evaluated for values of $\alpha_{\text {diff }}$ between 0.1 and 0.001 . In this scenario the detection performance depends only from $\alpha_{\text {diff }}$. The parameters of the simulated system are reported in Table II. The crosscorrelation function and the Receiver Operating Characteristic (ROC) curves have been considered to assess the detection perform of the proposed system. The ROC curves, shown in Fig. 2, are created by plotting the Probabilities of Detection $\left(\mathrm{P}_{\mathrm{D}}\right)$ against the desired Probabilities of False Alarm Rates $\left(\mathrm{DP}_{\mathrm{FA}}\right)$. Fig. 3 shows the values of the Probability of False Alarm Rate $\left(\mathrm{P}_{\mathrm{FA}}\right)$ vs $\mathrm{DP}_{\mathrm{FA}}$. The curves shown in Fig. 2 and in Fig. 3 are obtained at various

TABLE II: Simulated System Parameter

\begin{tabular}{clc}
\hline Parameters & Description & Values \\
\hline$f_{c}$ & Operating frequency. & $77 \mathrm{GHz}$ \\
\hline$M$ & Modulation order. & 2 \\
\hline$f_{\text {sep }}$ & Frequency separation. & $9 \mathrm{MHz}$ \\
\hline$N_{s}$ & Number of sampler per symbol. & 2 \\
\hline$F_{s}$ & Sampling Frequency. & $150 \mathrm{MHz}$ \\
\hline$\beta$ & Rolloff factor. & 0.95 \\
\hline$S$ & Filter Span in Symbol. & 12 \\
\hline$R_{s}$ & Output Samples per Symbol. & 12 \\
\hline$L$ & Length of Barker code. & 7 \\
\hline$T C$ & Training Cells. & 20 \\
\hline$G C$ & Guard Cells & 6 \\
\hline
\end{tabular}

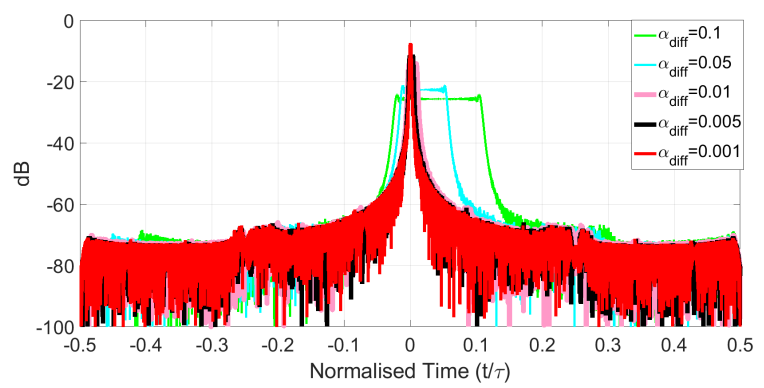

Fig. 4: Cross-Correlation between the main and the interference users for different values of $\alpha_{\text {diff. }}$.

threshold setting and for a specific SNR. The threshold is based on CA-CFAR [9], while the SNR is $-8 \mathrm{~dB}$. The $\mathrm{P}_{\mathrm{FA}}$ is calculated based on the number of false alarms and the number of trials while the $P_{D}$ is given from the number of detection on the number of trials. The number of Monte Carlo simulations used to obtain the results shown in Fig. 2 and in Fig. 3 is given from:

$$
N_{\text {trials }}=100 / \mathrm{DP}_{\mathrm{FA}}
$$

where $N_{\text {trials }}$ is the number of Monte Carlo runs that they are needed to obtain a $\mathrm{DP}_{\mathrm{FA}}$. The performance is evaluated for values of $\mathrm{DP}_{\mathrm{FA}}=10^{-4}, 10^{-3}, 10^{-2}$.

The cross-correlation function is obtained by calculating the mean value of 100 Monte Carlo runs for each $\alpha_{\text {diff }}$. Fig. 4 shows that as $\alpha_{\text {diff }}$ moves from 0.1 to 0.001 there is an intensity increase in the cross-correlation. High crosscorrelation can lead to problems such as ghost targets which will increase the number of false alarms and in undesirable losses of sensitivity for detecting radar targets. This behaviour is more evident by comparing the curves shown in Fig. 3 and in Fig. 2. In Fig. 3 it can be seen that for $\alpha_{\text {diff }} \in[0.1,0.005]$ the $P_{F A}$ is equal to $D P_{F A}$ while for $\alpha_{\text {diff }}=0.001$ the $\mathrm{P}_{\mathrm{FA}}$ is not equal to $\mathrm{DP}_{\mathrm{FA}}$. This is due to the fact that a ghost target generated by the interference is detected while it should be not. In Fig. 2 it can be seen that for $\alpha_{\text {diff }} \in[0.1,0.005]$ the $P_{D}$ move from 0.99 to 1 and the curves are very close to each other, while for $\alpha_{\text {diff }}=0.001$ the $\mathrm{P}_{\mathrm{D}}$ moves from 0.92 to 1 . The $P_{D}$ decreases because a ghost target arises due to the interference. When this ghost target falls in a the training cell, it causes a target masking which in turn lowers the $\mathrm{P}_{\mathrm{D}}$. Finally, from the simulated scenario it is observed that the minimum $\alpha_{\text {diff }}$ ensuring good detection performance is 0.005 .

\section{Communication Performance}

In this section, in order to evaluate the communication performance, the signal is assumed to experience a slowflat fading, therefore a time-invariant narrowband channel model is considered. Let $\mathbf{s}_{t x}$ be the vector which contains the transmitted signal samples. The received signal can be written as:

$$
\mathbf{s}_{r x}=\mathbf{h} \circ \mathbf{s}_{t x}+\mathbf{n}
$$




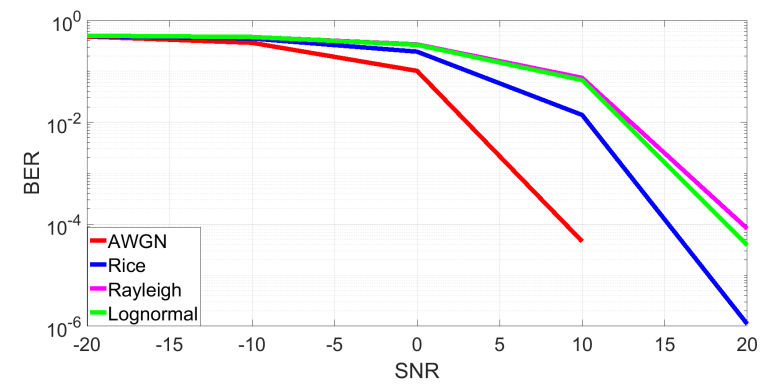

Fig. 5: Communication performance for four different channel models.

where $\mathbf{h}$ is the vector that contains the channel coefficients, $\mathbf{n}$ is the white Gaussian noise and the operator $\circ$ indicates the Hadamard, or entry-wise, product.

The complex elements of the vector $\mathbf{h}$ are drawn from a statistical distribution whose parameters depend on the propagation path. In additional to the Additive White Gaussian Noise (AWGN), the only scenario for which $\mathbf{h}=1$, three other cases are considered. For cases of which the Line of Sight (LOS) path is present, the channel is modelled as Rician with a Rice factor of $4 \mathrm{~dB}$. Conversely, when no LOS path exists, the channel coefficients $\mathbf{h}$ are drawn from a Rayleigh distribution with scale parameter $\sqrt{2} / 2$. Finally, in order to take into account shadowing and diffraction that can occur in urban environment, a combination of Rice and Lognormal is considered [11]. In this case the channel coefficients are obtained as the product of a Rice process normalised in power and a Lognormal variable, whose associated Gaussian variable has a standard deviation of 4 .

Assuming that the received signal has been equalised, the communication performance is evaluated for different values of SNR in terms of Bit Error Rate (BER), defined as the ratio between the number of bits wrongly decoded and the total number of bits sent. The curves are obtained from a Monte Carlo simulation during which $10^{7}$ bits are sent with order $\alpha=0.5$ and with SNR from -20 to $20 \mathrm{~dB}$. Fig. 5 shows the communication performance for four different channel models as: AWGN, Rice, Rayleigh and Lognormal. In rural environment where the main component is the LOS, the channel is modelled as a Rice, a BER $=10^{-4}$ is obtained with an SNR of $15 \mathrm{~dB}$. In urban environment, where the signal is subject to diffraction and shadowing and the channel is modelled as a Lognormal, to ensure a BER of $10^{-4}$ it is required to have SNR of some dBs more than in rural environment. Furthermore, where the LOS is not present and the channel is modelled as Rayleigh, a BER $=10^{-4}$ is obtained with an SNR of $20 \mathrm{~dB}$. Fig. 5 shows that in every environment the BER curves are very close to each others.

\section{CONCLUSIONS}

This paper presented the performance analysis of a system for joint radar and communication in automotive radar. The proposed system is evaluated for two neighbour vehicles in different environments. Simulation analysis showed that in the presence of two users it is possible to allocate them in the same frequency band, with small differences in the order of the FrFT. Additionally, the communication performance showed that this system can work in different environments ensuring good BER. Future developments include the performance evaluate of the proposed system in multi-user and multi target scenarios.

\section{ACKNOWLEDGMENT}

This work is supported by NXP Laboratories UK Limited and by the Engineering and Physical Sciences Research Council (EPSRC) Grant number EP/K014307/1 and the MOD University Defence Research Collaboration in Signal Processing.

\section{REFERENCES}

[1] C. Sturm, W. Wiesbech, "Waveform Design and Signal Processing Aspects for Fusion of Wireless Communications and Radar Sensing”, IEEE journals \& Magazines, vol.99, pp.1236-1259,2011.

[2] S. Patole, M. Torlak, D. Wang, and M. Ali, "Automotive Radars A review of signal processing techniques.", "Signal processing for smart vehicle technologies: part 2", IEEE Signal Processing Magazine", 2017.

[3] C. Clemente, I. Shorokhov, I. Proudler, J. Soraghan, "Radar Waveform Libraries Using Fractional Fourier Transform", 2014 IEEE Radar Conference, pp. 0855-0858, May 2014.

[4] D. Gaglione, C. Clemente, C. V. Ilioudis, A. R. Persico, I. Proudler, J. J. Soraghan, A. Farina, "Waveform design for communicating radar systems using Fractional Fourier Transform”, Digital Signal Processing, vol.80, pp. 57-69, September 2018.

[5] H. Rohling, Marc-M. Meinecke, "Waveform Design Principles for Automotive Radar Systems", Technical University of HamburgHarburg, Germany.

[6] P. Striano, C. Ilioudis, C. Clemente and J. Soraghan, "Communicating Radar Using FSK and Fractional Fourier Transform for Automotive Applications.",IET International Radar Conference 2018, 17-19 October 2018.

[7] F. Gini, A. De Maio and L. Patton, "Waveform Design and Diversity for Advanced Radar Systems.", IET Radar, Sonar and Navigation Series 22, 2012.

[8] V. Namias, "The Fractional Order Fourier Transform and its Application to Quantum Mechanics.", IMA Journal of Applied Mathematics, vol.25, no.3, pp.241-265, 1980.

[9] H. Rohling, "Radar CFAR thresholding in clutter and multiple target situation". IEEE Transactions on Aerospace and Electronic Systems, vol. AES-19, pp. 608-621, July 1983.

[10] F. Belloni, "Physical Layer Methods in Wireless Communication Systems". Helsinki University of Technology, SMARAD Centre of Excellence, 2014.

[11] G. Corazza, C. Ferrarelli, and F. Vatalaro, "A Rice-Lognormal Terrestrial and Satellite Channel Model", in 1994 Third Annual International Conference on Universal Personal Communications, San Diego, CA, USA, Sep. 1994, pp. 155-159. 